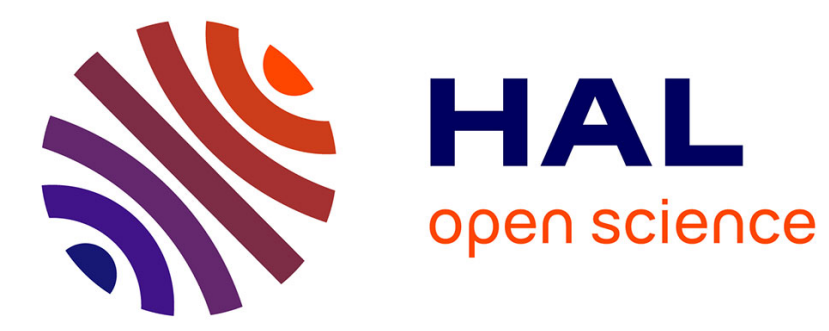

\title{
Monotonic Incompatibility Between Electing and Ranking
}

\author{
Michel L. Balinski, Andrew Jennings, Rida Laraki
}

\section{To cite this version:}

Michel L. Balinski, Andrew Jennings, Rida Laraki. Monotonic Incompatibility Between Electing and Ranking. 2008. hal-00339030

\section{HAL Id: hal-00339030 https://hal.science/hal-00339030}

Preprint submitted on 15 Nov 2008

HAL is a multi-disciplinary open access archive for the deposit and dissemination of scientific research documents, whether they are published or not. The documents may come from teaching and research institutions in France or abroad, or from public or private research centers.
L'archive ouverte pluridisciplinaire HAL, est destinée au dépôt et à la diffusion de documents scientifiques de niveau recherche, publiés ou non, émanant des établissements d'enseignement et de recherche français ou étrangers, des laboratoires publics ou privés. 


\title{
ECOLE POLYTECHNIQUE
}

Monotonic Incompatibility Between Electing and Ranking

\author{
Michel Balinski \\ Andrew Jennings \\ Rida Laraki
}

Novembre 2008

Cahier $n^{\circ}$ 2008-11

\section{DEPARTEMENT D'ECONOMIE}

Route de Saclay

91128 PALAISEAU CEDEX

(33) 169333033

http://www.enseignement.polytechnique.fr/economie/

mailto:chantal.poujouly@polytechnique.edu 


\title{
Monotonic Incompatibility Between Electing and Ranking
}

\author{
Michel Balinski ${ }^{1}$ \\ Andrew Jennings ${ }^{2}$ \\ Rida Laraki ${ }^{3}$
}

Novembre 2008

Cahier $n^{\circ}$ 2008-11

Résumé: $\quad$ Borda a proposé une méthode qui attribue des points à chacun des $m$ candidats. Condorcet a proposé une méthode qui attribue des points à chacun des différents $m$ ! classements des candidats. La première est plus appropriée pour élire. La seconde est plus appropriée pour classer. Chacune satisfait une certaine monotonie. Leurs monotonies sont incompatibles.

\begin{abstract}
Borda proposed a method that assigns points to each of the $m$ candidates. Condorcet proposed a method that assigns points to each of the $m$ ! rankings of candidates. The first is more appropriate for electing, the second is more appropriate for ranking. Each satisfies a different type of monotonicity. These monotonicities are incompatible.
\end{abstract}

Mots clés : $\quad$ Choix social, Borda, Condorcet, monotonie, incompatibilité, élire, classer.

Key Words : $\quad$ Social choice, Borda, Condorcet, monotonicity, incompatibility, electing, ranking.

Classification: $\quad$ JEL : D71, D02, D63. AMS : 91B14, 91B12.

\footnotetext{
${ }^{1}$ CNRS, Département d'Economie de l’Ecole Polytechnique, France.

${ }^{2}$ Department of Mathematics and Statistics, Arizona State University, USA.

${ }^{3}$ CNRS, Département d'Economie de l'Ecole Polytechnique, France.
} 


\title{
Monotonic incompatibility between electing and ranking*
}

\author{
Michel Balinski ${ }^{\alpha}$, Andrew Jennings ${ }^{\beta}$, and Rida Laraki ${ }^{\alpha}$ \\ ${ }^{\alpha}$ Laboratoire d'Econométrie de l'École Polytechnique and C.N.R.S., France. \\ ${ }^{\beta}$ Department of Mathematics and Statistics, Arizona State University, USA.
}

\begin{abstract}
Borda proposed a method that assigns points to each of the $m$ candidates. Condorcet proposed a method that assigns points to each of the $m$ ! rankings of candidates. The first is more appropriate for electing, the second is more appropriate for ranking. Each satisfies a different type of monotonicity. These monotonicities are incompatible.
\end{abstract}

\section{Introduction}

The traditional model of social choice assumes that each of $n$ judges or voters submits a rank-ordering of all the $m$ candidates. Two foremost questions are addressed: (1) the designation of a winner, (2) the designation of a ranking of all the candidates in the order of their merit.

The Chevalier de Borda's method [3] — first proposed by by Nicolaus Cusanus in 1433 [5] - assigns points to candidates. A voter contributes $k$ Borda-points to a candidate $C$ if $k$ candidates are ranked below $C$. The Borda-score of a candidate is the sum of her/his Borda-points. The Borda-winner is the candidate with the highest score. The Borda-ranking is established in descending order by the candidate's Borda-scores.

The Marquis de Condorcet [4] had an entirely different idea. A voter with the rank-order $\mathcal{Q}$ contributes $k$ Condorcet-points to a rank-order ${ }^{1} \mathcal{R}$ if the two rankings agree in $k$ pair-by-pair comparisons. The Condorcet-score of a ranking is the sum of its Condorcet-points over all voters. The Condorcet-ranking is the ranking with the highest score. ${ }^{2}$

By definition, the Chevalier and the Marquis addressed different questions.

Condorcet himself seems to have realized that the two ideas diverged, as Peyton Young [7] pointed out after a careful reading of his works. In short, Condorcet applied the idea of his "jury theorem" to the problem of ranking - find the maximum likelihood ranking given the inputs of individuals' rankings - but he seems to have seen that this did not pick out the maximum likelihood winner given the individuals' rankings. Young was the first to show unambiguously

\footnotetext{
* This work started during the visit of Andrew Jennings to the Ecole Polytechnique in 2008 and was partially supported by G.I.S. Sciences de la Décision.

${ }^{1}$ Note that there are $m$ ! rankings.

${ }^{2}$ This is the same rule suggested independently by John Kemeny [6].
} 
that finding society's winner is really different than finding society's ranking: the maximum likelihood approach applied to finding society's rank-order yields the Condorcet-ranking whereas applied to finding a winner it yields the Bordawinner. In fact, the divergence is even more fundamental, as is shown first by an example, second by a theorem.

The notation $A \succ B$ indicates that a voter ranks candidate $A$ ahead of candidate $B, A \approx B$ that she/he considers them tied, and $A \succeq B$ that $A \succ B$ or $A \approx B$. The symbols $\succ_{S}, \approx_{S}$, and $\succeq_{S}$ indicate society's ranking determined by a social decision method.

Consider the profile ${ }^{3}$

$$
\begin{gathered}
333: A \succ B \succ C \quad 333: B \succ C \succ A \quad 333: C \succ A \succ B \\
1: A \succ C \succ B,
\end{gathered}
$$

meaning, for example, that 333 voters have the ranking $A \succ B \succ C$.

The first 999 voters constitute a Condorcet-component: every candidate appears in every position exactly the same number of times. In the absence of the last voter, there is a clear tie between all three candidates - the perfect symmetry in the voter profile demands it. Accordingly, the last voter tips the scales and causes $A$ to be the winner and $B$ to be the loser. Borda's method yields this outcome.

However, $A \succ_{S} C \succ_{S} B$ is certainly not society's preferred ranking. Only one voter prefers it. 333 are directly opposed. There are two Condorcetrankings: $A \succ_{S} B \succ_{S} C$ and $C \succ_{S} A \succ_{S} B$. In each case 333 voters most prefer it, and both strictly dominate the Borda-ranking in the pair-by-pair comparisons. On the other hand, Condorcet's method yields a tie in winners, $A$ and $C$, which is unacceptable as well.

A method of ranking is: (1) winner-loser-unanimous if whenever all voters rank a candidate first (respectively, last) he/she is the winner (the loser); (2) choice-compatible if when all the voters rank a candidate first (respectively, last) and a Condorcet-component is added to the profile, then that candidate must be the winner (the loser); (3) rank-compatible if when a winner is removed from the set of candidates then the new ranking on the remaining candidates agrees with original ranking. ${ }^{4}$ Borda's method satisfies conditions (1) and (2) but not (3), whereas Condorcet's satisfies (1) and (3) but not (2).

Theorem 1 (Incompatibility [1, 2]). There is no method of ranking that is winner-loser-unanimous, choice- and rank-compatible for all preference-profiles (when there are at least three alternatives).

There is a third way to appreciate why there is a difference between designating winners and an order-of-finish. Borda asks as input messages rank-orders on candidates to determine society's winning candidate, instead of input messages of a single candidate (as was done in his era ... and is done in ours, with firstpast-the-post). Condorcet asks as input messages rank-orders on candidates to determine society's rank-order of candidates: but if society's rank-order is sought should not voters be asked as input messages the rank-orders over the $m$ ! rank-orders of all candidates [1, 2]? Condorcet's input messages of a single

\footnotetext{
${ }^{3}$ Originally in $[1]$

${ }^{4}$ Rank-compatibility is a weaker form of "local stability" [7].
} 
rank-order to determine society's rank-order is the counter-part of first-pastthe-post's input of a single candidate to determine society's winner. In short, the traditional model harbors a fundamental inconsistency in relating input messages to the outputs that are sought.

Another recurring and fundamental question in social choice is that of monotonicity. For a decision method to be just, a candidate must not be punished when he/she is ranked higher by one or more voters. There are many different conceptual formulations of monotonicity. The traditional formulation requires only that the winner remains a winner when she/he is ranked higher by some voters.

This paper shows that there is still another incompatibility that involves the Chevalier de Borda and the Marquis de Condorcet: each satisfies a different type of monotonicity, but no method satisfies both types of monotonicity.

\section{Choice-monotonicity}

Consider any pair of candidates $A$ and $B$ for which a method of ranking yields $A \succeq_{S} B$ in society's ranking. The method is choice-monotone if one or more voters move $A$ higher or $B$ lower implies $A \succ_{S} B$. Many social choice methods satisfy choice-monotonicity. This includes Borda's method, for if candidate $A$ has at least as many Borda points as $B$, then moving $A$ up will increase his Borda points while moving $B$ down cause his Borda points to decrease. In either case, $A$ will end up with more Borda points than $B$.

Condorcet's method is not choice-monotone (though it took some effort to find an example to prove it). Consider the following voter profile, $\mathcal{P}_{0}$ :

$$
\begin{aligned}
& k: A \succ B \succ C \succ D \succ E \\
& k: B \succ E \succ C \succ A \succ D \\
& 2: D \succ E \succ C \succ A \succ B \\
& 1: D \succ C \succ E \succ A \succ B \\
& 1: D \succ E \succ A \succ C \succ B
\end{aligned}
$$

If $k$ is at least 4 , then Condorcet's method will give a two-way tie for first between

$$
\mathcal{R}_{1}=A \succ_{S} B \succ_{S} C \succ_{S} D \succ_{S} E \quad \text { and } \quad \mathcal{R}_{2}=B \succ_{S} E \succ_{S} C \succ_{S} A \succ_{S} D .
$$

This can be checked easily enough with a computer, but if $k$ is at least 7 , a simple proof follows.

The relative pairwise rewards for one candidate preceding another in society's ranking are given in table 1 . For simplicity, the mean, $k+2$, has been subtracted from each element ${ }^{5}$. This decreases the Condorcet score of each ranking by $10(k+2)$, but does not affect the order of finish.

The rankings $\mathcal{R}_{1}$ and $\mathcal{R}_{2}$ both have a relative Condorcet-score of $5 k-10$, and it will be seen that no other ranking can have as high a score.

\footnotetext{
${ }^{5}$ For example, the reward of $A$ preceding $C$ is $k+1$ Condorcet points because in $k+1$ voters' rankings $A$ precedes $C$. After $k+2$ has been subtracted, this appears in the third column of the first row of the table as -1 .
} 


\begin{tabular}{|c|ccccc|}
\hline versus & $A$ & $B$ & $C$ & $D$ & $E$ \\
\hline$A$ & & 2 & -1 & $k-2$ & -2 \\
$B$ & -2 & & $k-2$ & $k-2$ & $k-2$ \\
$C$ & 1 & $-k+2$ & & $k-2$ & -1 \\
$D$ & $-k+2$ & $-k+2$ & $-k+2$ & & 2 \\
$E$ & 2 & $-k+2$ & 1 & -2 & \\
\hline
\end{tabular}

Table 1: Pairwise relative reward table for $\mathcal{P}_{0}$.

Any ranking which fails to preserve all five of the most significant pairwise relationships, $A \succ D, B \succ C, B \succ D, B \succ E$ and $C \succ D$, may score at most $3 k+2$ (the sum of at most $3 k-6$ from these five pairs and no more than 8 points from the other five pairs). For $k \geq 7$, this is less than $5 k-10$ so any ranking which doesn't satisfy these five significant relationships is excluded from winning. Out of the 120 possible societal rankings, there are 11 which meet this criterion, given in table 2 .

\begin{tabular}{|cccc|}
\hline$B C A D E$ & $B A E C D$ & $B A C E D$ & $B A C D E$ \\
$\mathcal{R}_{2}=B E C A D$ & $B E A C D$ & $B C E A D$ & $B C A E D$ \\
$A B C E D$ & $\mathcal{R}_{1}=A B C D E$ & $A B E C D$ & \\
\hline
\end{tabular}

Table 2: Remaining rankings after one step.

The chosen rankings $\mathcal{R}_{1}$ and $\mathcal{R}_{2}$ both have total contributions of 0 points from the eight less-significant pairwise relationships.

It is impossible for any ranking with $B \succ A, A \succ E$, and $C \succ E$ to achieve first place, as this would contribute $(-2)+(-2)+(-1)=-5$ Condorcet points, to which only 3 points could be added by the other two rankings. It is also impossible for any ranking with $A \succ E, E \succ D$, and $A \succ C$ to achieve first place, since this would also contribute $(-2)+(-2)+(-1)=-5$ Condorcet points, to which only 3 points could be added by the other two rankings. This eliminates 7 of the rankings, leaving those in table 3 .

$$
\begin{array}{|cc|}
\hline \mathcal{R}_{2}=B E C A D & B E A C D \\
B C E A D & \mathcal{R}_{1}=A B C D E \\
\hline
\end{array}
$$

Table 3: Remaining rankings after two steps.

Two are the chosen rankings. The Condorcet scores of the remaining two are computed below, where $\mathcal{C}(\mathcal{R})$ indicates the Condorcet score of ranking $\mathcal{R}$ (without the $5 k-10$ point contribution from the five most significant pairs), and $P_{X Y}$ indicates the pairwise relative reward for a ranking that places candidate $X$ above candidate $Y$.

$\mathcal{C}(B E A C D)=P_{B A}+P_{A C}+P_{E A}+P_{E C}+P_{E D}=(-2)+(-1)+2+1+(-2)=-2$

$\mathcal{C}(B C E A D)=P_{B A}+P_{C A}+P_{E A}+P_{C E}+P_{E D}=(-2)+1+2+(-1)+(-2)=-2$ 
Thus the two rankings $\mathcal{R}_{1}$ and $\mathcal{R}_{2}$ are tied for first with Condorcet's method and this profile of voters.

This profile is now used to show that Condorcet's method fails choicemonotonicity. First, create profile $\mathcal{P}_{1}$ from $\mathcal{P}_{0}$ by having one of the voters who ranked $C$ immediately above $A$ swap them. Since this would cause any ranking which placed $A$ above $C$ to increase by one point and any ranking which placed $C$ above $A$ to decrease by one point, Condorcet's method now gives a unique best societal ranking of $\mathcal{R}_{1}=A \succ_{S} B \succ_{S} C \succ_{S} D \succ_{S} E$.

Second, create profile $\mathcal{P}_{2}$ from $\mathcal{P}_{0}$ by having the voter who ranked $A$ immediately above $C$ swap them. Then Condorcet's method would give a unique best societal ranking of $\mathcal{R}_{2}=B \succ_{S} E \succ_{S} C \succ_{S} A \succ_{S} D$.

Thus, moving from profile $\mathcal{P}_{1}$ to $\mathcal{P}_{2}$ is accomplished by having two voters who placed $C$ immediately below $A$ swap them, and this causes society's preferred ranking to change from

$$
\mathcal{R}_{1}=A \succ_{S} B \succ_{S} C \succ_{S} D \succ_{S} E \quad \text { to } \quad \mathcal{R}_{2}=B \succ_{S} E \succ_{S} C \succ_{S} A \succ_{S} D .
$$

So the societal ranking has changed from $C \succ_{S} E$ to $E \succ_{S} C$ when $C$ was moved strictly higher in two voters' rankings.

\section{Rank-monotonicity}

A method is rank-monotone if when one or more voters rank the winner higher society's ranking remains the same (not only the winner remains the same). This requirement is quite selective, rejecting many methods, including Borda's ${ }^{6}$.

Condorcet's method satisfies rank-monotonicity. For if a candidate is first in the Condorcet-ranking $\mathcal{R}$ and one voter ranks that candidate higher by one position, then $\mathcal{R}$ receives one more Condorcet-point whereas the other rankings either gain one point or lose one point, so the ranking $\mathcal{R}$ must remain uniquely in first place.

Caution is required here, since Condorcet's method may give a tie among several rankings instead of one unique societal ranking. As such, Condorcet's method doesn't even fit the strict definition of a ranking function: it does not have an unrestricted domain. Yet in all cases where Condorcet's method gives a clear winning candidate (all chosen societal rankings have the same candidate in first place), one or more voters ranking the winning candidate higher will not change the set of societal rankings chosen, so it is reasonable to claim that Condorcet's method satisfies rank-monotonicity.

Thus rank-monotonicity is enjoyed by Condorcet-rankings but not Bordarankings.

\section{Incompatibility}

Rank-monotonicity and choice-monotonicity are not compatible.

\footnotetext{
${ }^{6}$ In the profile $3: A \succ B \succ C$ and $2: C \succ B \succ A$, the candidates $A, B$, and $C$ receive 6,5 , and 4 Borda points respectively, giving the societal ranking $A \succ_{S} B \succ_{S} C$. If the two voters who rank $A$ last were to raise him one position, this would reward $A$ with two Borda points at the expense of $B$, giving a societal outcome of $A \succ_{S} C \succ_{S} B$.
} 
Theorem 2. There is no ranking-function that is impartial, unanimous, rankand choice-monotone (when there are at least three candidates and at least two voters).

Proof. Let $2 k+i$ equal the number of voters, with $i$ either 0 or 1 , and $\mathcal{P}$ be the profile

$$
\begin{gathered}
k: A \succ B \succ C \succ A_{1} \succ \cdots \succ A_{n} \quad k: B \succ C \succ A \succ A_{1} \succ \cdots \succ A_{n} \\
i: A \approx B \approx C \succ A_{1} \succ \cdots \succ A_{n} .
\end{gathered}
$$

By impartiality, the profile

$$
\begin{gathered}
k: B \succ A \succ C \succ A_{1} \succ \cdots \succ A_{n} \quad k: B \succ C \succ A \succ A_{1} \succ \cdots \succ A_{n} \\
i: A \approx B \approx C \succ A_{1} \succ \cdots \succ A_{n}
\end{gathered}
$$

implies $A \approx_{S} C$. The profile $\mathcal{P}$ is obtained when the first $k$ voters move $A$ above $B$. By choice-monotonicity, profile $\mathcal{P}$ must imply $A \succ_{S} C$.

Similarly, the profile

$$
\begin{gathered}
k: A \succ B \succ C \succ A_{1} \succ \cdots \succ A_{n} \quad k: B \succ A \succ C \succ A_{1} \succ \cdots \succ A_{n} \\
i: A \approx B \approx C \succ A_{1} \succ \cdots \succ A_{n}
\end{gathered}
$$

implies $A \approx_{S} B$ and changes into profile $\mathcal{P}$ when the second group of voters move $C$ above $A$. Thus the profile $\mathcal{P}$ must imply $B \succ_{S} A$. Unanimity now determines the complete outcome for $\mathcal{P}$ to be $B \succ_{S} A \succ_{S} C \succ_{S} A_{1} \succ_{S} \cdots \succ_{n}$.

By rank-monotonicity, the profile

$$
\begin{gathered}
k: B \succ A \succ C \succ A_{1} \succ \cdots \succ A_{n} \quad k: B \succ C \succ A \succ A_{1} \succ \cdots \succ A_{n} \\
i: A \approx B \approx C \succ A_{1} \succ \cdots \succ A_{n},
\end{gathered}
$$

must imply the same outcome as $\mathcal{P}$, including $A \succ_{S} C$, which contradicts the earlier impartiality result $\left(A \approx_{S} C\right)$ for this profile.

This proof admits inputs with equivalents: they are not strict rank-orders. The same proof is valid for strict rank-orders as inputs when $i=0$.

\section{Condorcet's monotonicity violations}

The example which shows Condorcet's method violates choice-monotonicity includes five candidates. What is the minimum number of candidates necessary to create such a profile?

In fact, three or four candidates is too few.

Three candidates. Any violation of choice-monotonicity would be a pair of profiles where the unique winning ranking goes from

$$
A_{1} \succ_{S} A_{2} \succ_{S} A_{3} \quad \text { to } \quad A_{3} \succ_{S} A_{2} \succ_{S} A_{1}
$$

when $A_{2}$ is ranked higher by one or more voters. But when $A_{1} \succ_{S} A_{2} \succ_{S} A_{3}$ is the unique winner, it must have more Condorcet-points than $A_{1} \succ_{S} A_{3} \succ_{S} A_{2}$. 
After some voters have ranked $A_{2}$ higher, this requires that $A_{2} \succ_{S} A_{3} \succ_{S} A_{1}$ have more Condorcet-points than $A_{3} \succ_{S} A_{2} \succ_{S} A_{1}$.

Four candidates. Suppose Condorcet's method violates choice-monotonicity with four candidates. Then there is a profile of voters, $\mathcal{P}_{0}$ which gives a unique best ranking of

$$
A_{1} \succ_{S} A_{2} \succ_{S} A_{3} \succ_{S} A_{4},
$$

and another profile of voters, $\mathcal{P}_{1}$ with either:

(i) The only difference between $\mathcal{P}_{1}$ and $\mathcal{P}_{0}$ is that some voters rank $A_{2}$ higher, but Condorcet's method applied to $\mathcal{P}_{1}$ gives a unique best ranking that has $A_{3} \succ_{S} A_{2}$ or $A_{4} \succ_{S} A_{2}$.

(ii) The only difference between $\mathcal{P}_{1}$ and $\mathcal{P}_{0}$ is that some voters rank $A_{3}$ higher, but Condorcet's method applied to $\mathcal{P}_{1}$ gives a unique best ranking with $A_{4} \succ_{S} A_{3}$.

$A_{1}$ could not be the subject of a violation of choice-monotonicity because, since Condorcet's method is rank-monotone, one or more voters ranking $A_{1}$ higher cannot change society's ranking. $A_{4}$ could not be the subject of such a violation because he doesn't defeat any candidates in $\mathcal{P}_{0}$. Thus the two cases above are exhaustive.

Case (i): Here there are several strings of candidates which, if they appear together in the winning ranking of $\mathcal{P}_{1}$, must appear in the same order as in the winning ranking of $\mathcal{P}_{0}$. For example, $A_{2}$ can not appear immediately after $A_{3}$ because $A_{1} \succ_{S} A_{2} \succ_{S} A_{3} \succ_{S} A_{4}$ defeats $A_{1} \succ_{S} A_{3} \succ_{S} A_{2} \succ_{S} A_{4}$ in $\mathcal{P}_{0}$ so the number of Condorcet-points awarded must be greater for $A_{2} \succ_{S} A_{3}$ than it is for $A_{3} \succ_{S} A_{2}$. But this must hold in $\mathcal{P}_{1}$ as well. Thus any ranking with $A_{2}$ immediately following $A_{3}$ will have strictly fewer Condorcet-points than the same ranking with these two candidates reversed.

The other such strings of candidates are $A_{3} A_{4}$ and $A_{2} A_{3} A_{4}$. This leaves the possible winning rankings in table 4 .

$$
\begin{aligned}
& A_{3} \succ_{S} A_{1} \succ_{S} A_{2} \succ_{S} A_{4} \\
& A_{3} \succ_{S} A_{1} \succ_{S} A_{4} \succ_{S} A_{2} \\
& A_{3} \succ_{S} A_{4} \succ_{S} A_{1} \succ_{S} A_{2} \\
& A_{4} \succ_{S} A_{1} \succ_{S} A_{2} \succ_{S} A_{3} \\
& A_{4} \succ_{S} A_{2} \succ_{S} A_{1} \succ_{S} A_{3}
\end{aligned}
$$

Table 4: Possible winning rankings

It is also necessary that the winning ranking have $A_{2} \succ_{S} A_{1}$ in order for it to increase in points more than $A_{1} \succ_{S} A_{2} \succ_{S} A_{3} \succ_{S} A_{4}$ in moving from $\mathcal{P}_{0}$ to $\mathcal{P}_{1}$. This leaves $A_{4} \succ_{S} A_{2} \succ_{S} A_{1} \succ_{S} A_{3}$ as the only possible winning ranking for $\mathcal{P}_{1}$, which will be examined below.

Case (ii): In the same manner as above, the unique best ranking in $\mathcal{P}_{1}$ cannot contain $A_{4} \succ_{S} A_{3}$ contiguously. And rankings without $A_{3} \succ_{S} A_{1}$ or $A_{3} \succ_{S} A_{2}$ are eliminated because they are unable to overtake the winner from $\mathcal{P}_{0}$ in moving to $\mathcal{P}_{1}$. This leaves only $A_{4} \succ_{S} A_{1} \succ_{S} A_{3} \succ_{S} A_{2}$ and $A_{4} \succ_{S} A_{2} \succ_{S} A_{3} \succ_{S} A_{1}$ as possible winning rankings.

In any case, the sum of the Condorcet points awarded in $\mathcal{P}_{0}$ for a ranking having $A_{1} \succ_{S} A_{4}, A_{2} \succ_{S} A_{4}$ and $A_{3} \succ_{S} A_{4}$ must be positive since $A_{1} \succ_{S}$ $A_{2} \succ_{S} A_{3} \succ_{S} A_{4}$ defeats $A_{4} \succ_{S} A_{1} \succ_{S} A_{2} \succ_{S} A_{3}$. The Condorcet contribution 
from these three pairwise comparisons in $\mathcal{P}_{1}$ must be at least that of $\mathcal{P}_{0}$, hence positive. This means that the unique winning ranking cannot have $A_{4}$ in first place, because it could gain Condorcet-points merely by moving $A_{4}$ from first to last place.

This eliminates the only rankings from either case (i) or case (ii) which remained as violations of choice-monotonicity, so proves that a violation of choicemonotonicity is not possible with four candidates.

This does not mean that Condorcet's method satisfies choice-monotonicity with three and four candidates. In fact, such a statement wouldn't even make sense with the restricted domain of Condorcet's method. These results only prove that, with three or four candidates, it is impossible to find a violation of choice-monotonicity from the domain of voter profiles where Condorcet's method gives a unique best societal ranking.

Condorcet's method could be extended into a true ranking function with the addition of a tie-breaking rule that selects among society's rankings when there are ties. Naturally, this would strip the method of its impartiality, but it may also cause the method to fail choice- or rank-monotonicity, in accord with the monotonicity theorem above.

\section{More monotonicity}

A method is rank-order-monotone if a candidate does not lower in society's ranking when he/she is ranked higher by one or more voters.

Condorcet's method fails this version of monotonicity also. Take the voter profile, $\mathcal{P}_{0}$ :

$$
\begin{array}{ll}
k: A \succ B \succ C \succ D \succ E \succ F & k: B \succ E \succ F \succ C \succ A \succ D \\
1: F \succ D \succ E \succ C \succ A \succ B & 1: F \succ D \succ A \succ C \succ E \succ B \\
1: D \succ C \succ E \succ A \succ F \succ B & 1: D \succ E \succ F \succ C \succ A \succ B .
\end{array}
$$

If $k$ is at least 4 , then Condorcet's method will give a two-way tie for first between

$$
A \succ_{S} B \succ_{S} C \succ_{S} D \succ_{S} E \succ_{S} F \quad \text { and } \quad B \succ_{S} E \succ_{S} F \succ_{S} C \succ_{S} A \succ_{S} D \text {. }
$$

A manipulation similar to the one above will give a pair of profiles where two voters moving $C$ above $A$ will cause the preferred societal ranking to go from

$$
A \succ_{S} B \succ_{S} C \succ_{S} D \succ_{S} E \succ_{S} F \quad \text { to } \quad B \succ_{S} E \succ_{S} F \succ_{S} C \succ_{S} A \succ_{S} D \text {. }
$$

This violation requires six candidates - five is insufficient. A proof of this fact follows the same lines as the above proof that it is not possible to find a violation of choice-monotonicity with four candidates.

\section{$7 \quad$ Stronger incompatibilities?}

Theorem 2 requires impartiality, a standard requirement for any real voting system, but a quite strict requirement in theoretical social choice. Can this requirement be weakened? 
If the domain is restricted to prohibit ties in the voters' input, it is tempting to postulate that every ranking function that is unanimous, choice-monotone, and rank-monotone is equivalent to a dictatorship (with some appropriate minimum on the number of candidates).

In fact, this is false. Consider the method where, for $n$ candidates, a sequence of "decisive" voters, $v_{1}, v_{2}, \ldots, v_{n-1}$, is chosen. $v_{1}$ 's winner is society's winner; $v_{2}$ 's preferred candidate of the remaining candidates is society's second choice; and so on.

This is reminiscent of the way sports teams are often chosen (in schoolyards, community gyms, and professional drafts). Two or more coaches or captains are chosen, and they, in turn, draft from a pool of available candidates.

This method is equivalent to a dictatorship if and only if $v_{1}=v_{2}=\cdots=$ $v_{n-1}$. It is unanimous, choice-monotone, and rank-monotone for any number of candidates.

Unanimity and rank-monotonicity are easy to verify. For choice-monotonicity, note that a voter moving a candidate up will either not affect which candidate is most preferred by the voter, or will change it to the candidate who was moved up. Thus, when one or more voters move a candidate up, it may cause that candidate to win in an earlier round, but same string of candidates who are ahead of the candidate in the new societal ranking must be the winning string of candidates in the old ranking, so it is clear that the candidate who was moved up can not be ranked lower than any candidates he defeated before.

This method is, in fact, impartial with respect to candidates, though not to voters. There is another method that is impartial with respect to voters but not to candidates.

Consider the ordered set of candidates $A_{1}, A_{2}, \ldots, A_{n}$, and the choice rule that chooses $A_{i}$ as the winner if $A_{1}, \ldots, A_{i-1}$ are not ranked first by any voters and $A_{i}$ is ranked first at least once. ( $A_{1}$ will win if he is the favorite of any voter and $A_{n}$ will win only if he is the favorite of all voters.)

After a winner is chosen, repeatedly applying the rule to the remaining field will choose second-place, third-place, etc., an entire societal ranking. This method of generating a societal ranking is unanimous, rank- and choice-monotone for any number of candidates.

It is unanimous because if every voter places candidate $A$ ahead of candidate $B$, then candidate $B$ cannot be chosen until $A$ is eliminated from considerationthat is, $A$ must place higher than $B$.

It is rank-monotone because if $A_{i}$, society's first placed candidate is ranked higher by one or more voters, he/she will still be ranked first by at least one voter and $A_{1}, \ldots, A_{i-1}$ will still not be ranked first by any voters. This candidate is then eliminated from all further calculations so the remainder of the societal ranking must be independent of the voters' rankings of this candidate.

It remains to be shown that this method is choice-monotone.

First note that if $A_{1}$ is excised from all voter profiles, society's ranking among the remaining candidates remains the same. (This only holds for $A_{1}$, the most advantaged candidate, not for all candidates.) To see this, let $\mathcal{P}_{0}$ be a profile that includes $A_{1}$ and $\mathcal{P}_{1}$ be the same profile without $A_{1}$. In any round before $A_{1}$ is chosen in $\mathcal{P}_{0}, A_{1}$ must not be ranked first by any voters, so the set of voters' first-placed candidates must be the same in $\mathcal{P}_{0}$ and $\mathcal{P}_{1}$ and the same candidate must be chosen in $\mathcal{P}_{0}$ and $\mathcal{P}_{1}$. After $A_{1}$ is chosen, he is dropped from consideration, so the remainder of the rankings must be the same in $\mathcal{P}_{0}$ and $\mathcal{P}_{1}$. 
Now let $\mathcal{P}_{0}$ be a profile with $A_{i} \succ_{S} A_{j}$ and $\mathcal{P}_{1}$ be identical to $\mathcal{P}_{0}$ with $A_{i}$ moved higher by one or more voters. If $i<j$ then it may be assumed that $i=1$ since any more advantaged candidates could be dropped without affecting the relative societal ranking for $A_{i}$ and $A_{j}$. In any given round, $A_{i}$ being moved higher by one or more voters can only affect the set of voters' top choices by moving into that set, at which point it would win the round, so $A_{i}$ can move up in society's ranking with $\mathcal{P}_{1}$, but cannot affect any earlier rounds, so $A_{i}$ cannot lose to someone he defeated in $\mathcal{P}_{0}$.

If $i>j$, consider the round in $\mathcal{P}_{0}$ where $A_{i}$ wins. All of the voters' top choices must be $A_{i}$ or less advantaged than $A_{i}$, so every voter must have $A_{i}$ or someone less advantaged than $A_{i}$ ranked above $A_{j}$. With $\mathcal{P}_{1}$, when $A_{i}$ is moved up by some voters, this is still true. If $A_{j} \succ_{S} A_{i}$ in $\mathcal{P}_{1}$, then there must be at least one candidate less advantaged than $A_{i}$ who defeats $A_{j}$ (and hence $A_{i}$ ) in society's ranking, otherwise $A_{j}$ could not be any voter's preferred candidate. But this contradicts the fact proved above, that $A_{i}$ cannot lose in $\mathcal{P}_{1}$ to any less advantaged candidates which he defeated in $\mathcal{P}_{0}$.

\section{References}

[1] Michel Balinski and Rida Laraki, "A theory of measuring, electing and ranking," Proceedings of the National Academy of Sciences, USA 104 (2007) $8720-8725$.

[2] Michel Balinski and Rida Laraki, One-Value, One-Vote: Measuring, Electing, and Ranking (tentative title), to appear 2009.

[3] Le Chevalier Jean-Charles de Borda, "Mémoire sur les élections au scrutin," Histoire de l'Académie royale des sciences (1784) 657-665. A footnote states that the ideas were presented before the Academy on June 16, 1770.

[4] Le Marquis de Condorcet, Essai sur l'application de l'analyse à la probabilité des décisions rendues à la pluralité des voix, Paris, 1785, l'Imprimerie royale.

[5] Gunter Hägele and Friedrich Pukelsheim, "The electoral systems of Nicolaus Cusanus," to appear in Hg. G. Christianson, T.M. Izbicki, and C.M. Bellitto, eds., The Church, the Councils and Reform: Lessons from the Fifteenth Century, Catholic University of America Press, Washington, DC.

[6] John Kemeny, "Mathematics without numbers," Daedalus 88 (1959) 571591; or chapter II, "Preference rankings: an axiomatic approach," in J. G. Kemeny and J. L. Snell, Mathematical Models in the Social Sciences, Ginn and Co., 1962.

[7] H. P. Young, "Condorcet's theory of voting," American Political Science Review 82 (1988) 1231-1244. 